\title{
Subtrochanteric Fracture as First Sign of Metastatic Breast Cancer: Case Presentation
}

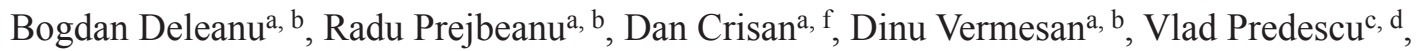 \\ Mihaela Iacob ${ }^{\mathrm{e}}$, Alis Dema ${ }^{\mathrm{a}}$
}

\begin{abstract}
Atraumatic subtrochanteric fractures represent a serious injury of the lower limb as they represent the clinical expression of a preexisting pathology. Atypical subtrochanteric femur fractures can result from long-term bisphosphonate therapy but a primary or metastatic tumor of the proximal femur should always be included in the differential diagnosis. We present the case of a young female patient without any previous pathological conditions that presented to the emergency room with a subtrochanteric fracture. She was admitted to our clinic and treated with a long cephalomedullary device (CMD). Tissue from the fracture zone was harvested and sent for a pathology analysis. While pathologic bone fractures are not a new entity by any mean in orthopedic practice, they seldom are the first sign of a metastatic disease. This presents a therapeutic challenge especially in young patients because of the reserved overall prognosis in the medium and long term. Better screening methods should be employed in a bid to eliminate this category of patients. We consider this case of a young woman with undetected breast cancer and one skeletal-related event (SRE) in the form of a pathological bone subtrochanteric fracture as an interesting development of the underlying pathology since as the literature shows these cases are quite rare and she did not present any of the usual symptoms associated with a bone metastasis.
\end{abstract}

Keywords: Subtrochanteric; Fracture; Bone; Metastasis; Breast cancer

Manuscript accepted for publication June 30, 2015

a"Victor Babes" University of Medicine and Pharmacy, 2 Eftimie Murgu Sq., 300041 Timisoara, Romania

b“Pius Branzeu” Emergency County Clinical Hospital Timisoara, 1st Orthopedics and Traumatology Clinic, 10 I. Bulbuca Blvd., 300737 Timisoara, Romania

"Carol Davila" University of Medicina and Pharmacy, 8 Eroii Sanitari Blvd., 050474 Bucharest, Romania

d“St. Pantelemon" Emergency Clinical Hospital Bucharest, Orthopedics and Traumatology Clinic 340 - 342 Pantelimon Road, Sector 2, 033092 Bucharest, Romania

"Pius Branzeu" Emergency County Clinical Hospital Timisoara, Pathology Department 10 I. Bulbuca Blvd., 300737 Timisoara, Romania

fCorresponding Author: Dan Crisan, "Victor Babes" University of Medicine and Pharmacy, 2 Eftimie Murgu Sq., 300041 Timisoara, Romania.

Email: crisan.dan@gmail.com

doi: http://dx.doi.org/10.14740/jmc2216w

\section{Introduction}

Atraumatic fractures of the proximal femur are defined as those fractures that occur without any kind of trauma at the level of the hip, usually appearing when the patients step on the respective lower limb, sometimes under a load of some sort or when they climb up or down some steps. These include bumping into a piece of furniture, but they exclude fractures caused by same height falls or those that are produced by a fall from height.

Because of the extremely low energy involved the underlying previous bone pathology is essential, meaning that the fractures would not occur on healthy bone. The most prevalent disease affecting bone structure is osteoporosis and indeed osteoporotic fractures of the proximal femur are an epidemiological problem. But the vast majority of proximal femur fractures on osteoporotic bone require a minimal trauma such as a fall from the same height. A different but related entity is atraumatic subtrochanteric fractures associated with long-term bisphosphonate therapy [1-3] but there are also some recent studies that question this association [4].

Malignancies either primary or metastatic represent another principal cause of pathological bone fracture and they should be suspected in all cases where there is no history of trauma involved in a fracture, especially if one of the long bones is involved. Other causes, such as stress fractures can usually be ruled out by taking an accurate history.

\section{Case Report}

We present the case of a 50-year-old woman without any previously known pathologies that presented to the emergency room accusing debilitating pain in her left hip with an acute onset in the previous $2 \mathrm{~h}$ that was caused by bumping into a piece of furniture. She was non-weight-bearing, the hip was extremely painful on palpation and mobilization, and the left lower limb was externally rotated by approximately $30^{\circ}$ and shortened by approximately $3 \mathrm{~cm}$. The patient had a height of $157 \mathrm{~cm}$ and a weight of $57 \mathrm{~kg}$. She was a non-smoker, occasional alcohol drinker and denied the regular use of other drugs. Medical history revealed no serious conditions. A standard emergency pelvic X-ray (Fig. 1) was performed and to evaluate the extent of the lesion, a CT 


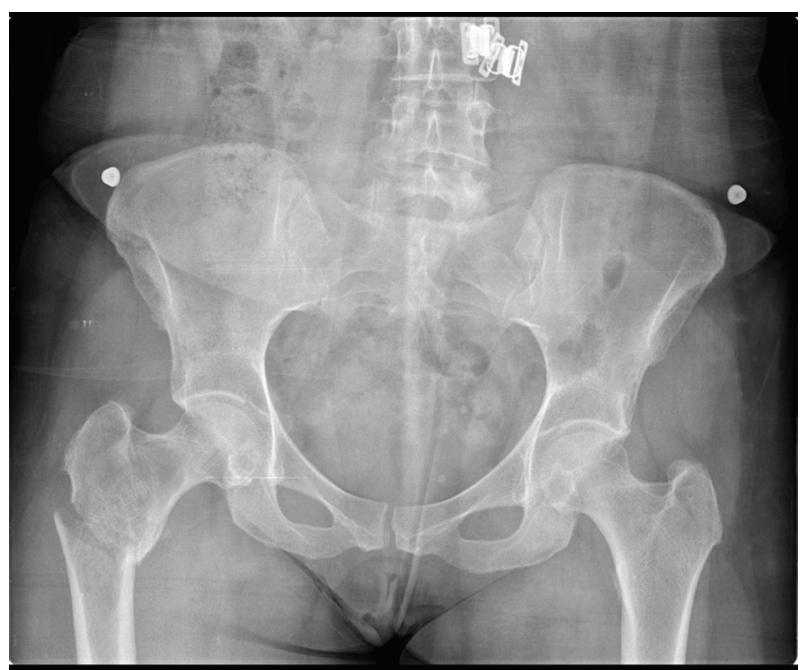

Figure 1. Pelvic AP X-ray showing a Seinsheimer IIB pathological bone subtrochanteric fracture.

scan with three-dimensional (3D) reconstruction was also obtained (Fig. 2).

After a thorough preoperative examination that revealed no clinically manifest alterations, she was treated surgically with reamed intramedullary nailing (Fig. 3) with a long cephalomedullary device (CMD) (Gamma 3 Long, Stryker, Kalamazoo, MI, USA). During the surgery biopsy material was collected from the fracture site during the drilling of the femoral neck tunnel and sent to analysis at our hospitals Pathology Department.

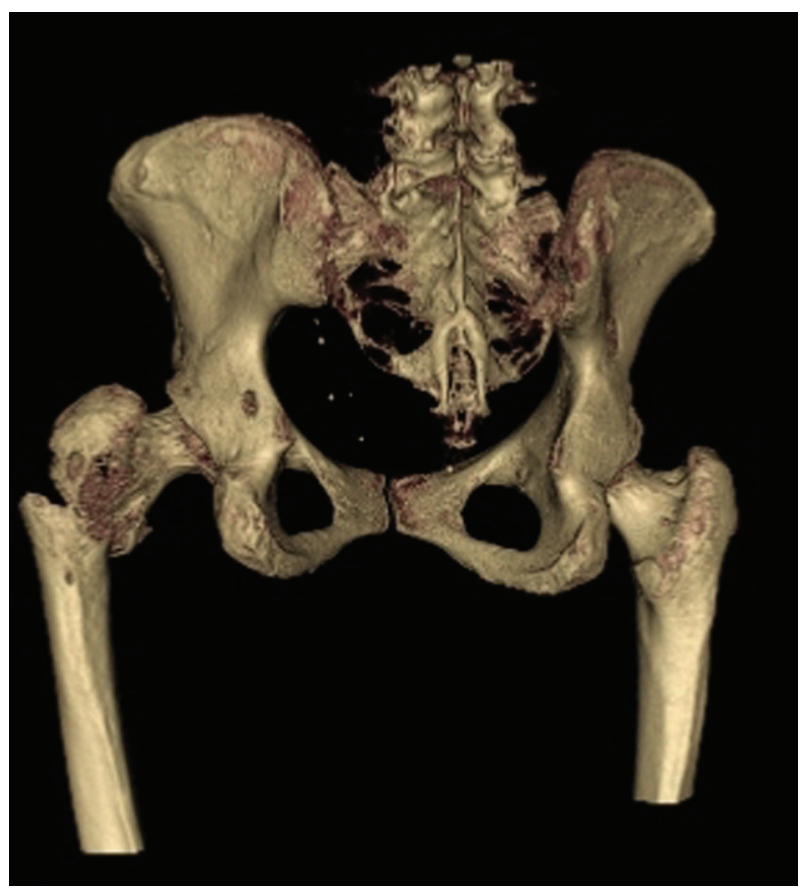

Figure 2. 3D CT scan showing a Seinsheimer IIB pathological bone subtrochanteric fracture with secondary dissemination in the trochanteric and femoral neck region.

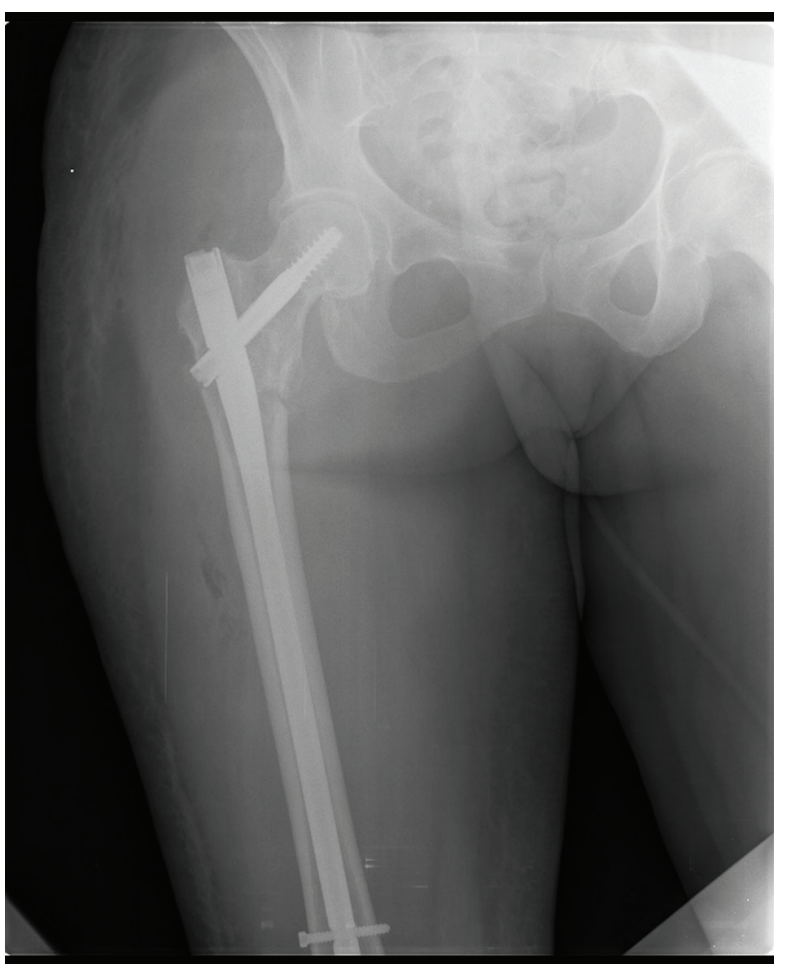

Figure 3. Postoperative hip X-ray showing intralesional fixation with a long CMD.

\section{Morphological method}

The fragments of bone biopsy were prepared by the usual technique: 24 -h fixation in $10 \%$ formalin solution, paraffin inclusion, sectioning in 3 - $5 \mu$ slices. For the routine morphological study, the classic hematoxylin-eosin (H\&E) method, standard technique, was used. We established the microscopic lesion diagnosis on the slides stained with the usual method.

\section{Immunohistochemistry (IHC) methods}

Detecting the origin of the metastatic lesion involved IHC determinations with the following antibodies: anti-cytokeratin 7 (CK7), anti-p63, anti-mammaglobin, anti-E-cadherin, and anti-estrogen receptor (ER) $[5,6]$. Additional sections from the paraffin blocks were placed on specially treated slides to insure a better adherence during the antigenic restoration process.

The primary antibodies used for the IHC investigation are shown in Table 1.

The NOVOLINK polymeric detection system was used, and the final reaction product was visualized with diaminobenzidine (DAB), considering that the nuclei were colored with hematoxylin.

\section{Discussion}

On the slides with the usual H\&E stain, we identified osseous 
Table 1. Antibody Characteristics

\begin{tabular}{llllll}
\hline Antibody & Clone & Manufacturer & Pretreatment & Dilution \\
\hline CK7 & OVTL & NOVOCASTRA & HIER-99 ${ }^{\circ} \mathrm{C}$ in Dako Target Retrieval solution, $\mathrm{pH} 6$ & $1 / 50$ \\
p63 & - & DAKO & HIER-99 ${ }^{\circ} \mathrm{C}$ in Dako Target Retrieval solution, pH 6 & $1 / 50$ \\
E-cadherin & NCH-38 & DAKO & HIER-99 ${ }^{\circ} \mathrm{C}$ in Dako Target Retrieval solution, $\mathrm{pH} 6$ & RTU \\
Mamaglobin & $304-\mathrm{IA} 5$ & DAKO & HIER-99 ${ }^{\circ} \mathrm{C}$ in Dako Target Retrieval solution, $\mathrm{pH} 6$ & $1 / 100$ \\
ER & 1D5 & DAKO & HIER-99 ${ }^{\circ} \mathrm{C}$ in Dako Target Retrieval solution, $\mathrm{pH} 6$ & $1 / 50$ \\
\hline
\end{tabular}

and periosteal tissue of various sizes intertwined with tumor cell groups of an apparent epithelial source (Fig. 4) with focal appearances of glandular lumens (Fig. 5); the tumor cells have a polygon aspect, eosinophil cytoplasm, evident cell limits, and moderated nuclear pleomorphism (Fig. 6). The morphological characteristics were deemed suggestive for the mammary origin of the proliferation which was confirmed by the IHC profile of the tumor cells: diffuse and intense reactivity for CK7 9 (Fig. 7), mammaglobin (Fig. 8), E-cadherin (Fig. 9), ER (Fig. 10) and reactivity restricted to isolated cells for p63, with a greater number of positive nuclei of stromal fusiform cells (Fig. 11).

\section{Conclusion}

Breast cancer is the leading cause of cancer deaths in females living in less developed countries with an estimate of 1.7 million new cases worldwide in 2012 and 521,900 deaths [7].

Metastatic dissemination is a frequent event in the clinical course of breast carcinoma. The site of the first metastasis was skeletal in $46 \%$ of the 648 patients with metastatic breast carcinoma studied by Solomeyer et al. Bone metastases are rarely asymptomatic, pain being a frequent clinical sign. Skeletalrelated events (SREs) frequently complicate a bone metastasis and depending on the location of the dissemination they can represent spinal compressions, pathological bone fractures,

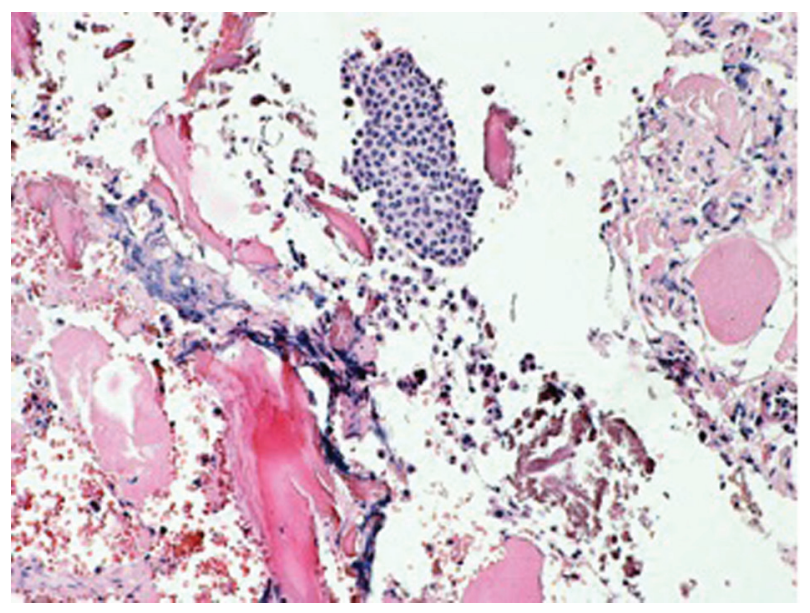

Figure 4. H\&E stain, $\times 100$ : a solid group of tumor cells surrounded by areas of bone tissue, connective tissue, a smooth muscle cell and extravased red blood cells, (B). bone marrow infiltration or malignancy hypercalcemia [8]. Secondary bony determinations are characterized by osteolytic lesions produced by pathologically activated osteoclasts [9]. This activity usually causes a substantial amount of pain, creating the premises for diagnosing the tumor.

Substantial financial resources are used for treating SREs. A Danish study by Svendsen et al on 569 breast patient cases with bone metastases from the 2003 - 2009 period found that significant resources were used in the post-SRE period for spinal cord compressions and in the diagnostic period of pathological fractures. Their study found only 153 patients with only one SRE and a pathological fracture was recorded in only 19 out of those, without specification on the level of the fracture [10]. Their findings were supported by previous studies from Spain and Portugal [11, 12]. Another longer term (1999 - 2007) Danish study found the mean age of the one SRE breast cancer patient with bony metastases to be of 67.4 years (range, 25 - 95 years) with a high incidence of SRE in the year after the diagnosis of the bone metastasis [13]. Wegener et al found that $98 \%$ of their patients presented with pain and $63 \%$ had a pathological fracture and their mean age at surgery was 57.3 years (range: 30.6 - 83.6 years) and 60 out of 115 patients received an intralesional implant [14].

There are several ways to decrease the risk of developing breast cancer such as maintaining a healthy lifestyle with a $\mathrm{BMI}$ in the normal range, physical activities and cutting back on alcohol. Early diagnosis is paramount, and clinical examination and awareness of clinical signs and symptoms com-

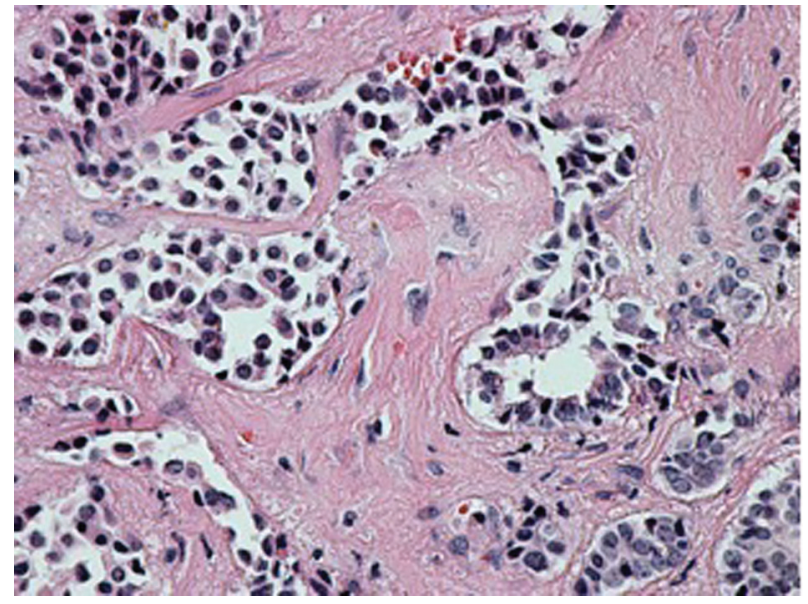

Figure 5. H\&E stain, $\times 200$ solid group of cohesive tumor cells with moderated pleomorphic nuclei surrounded by RBCs. 


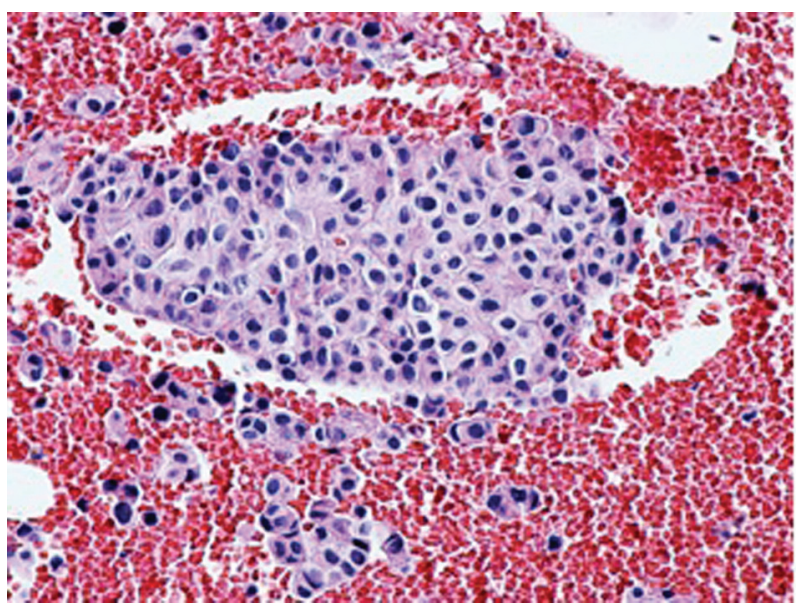

Figure 6. H\&E stain, $\times 400$ Glandular lumen revealed in one of the tumor cell nests

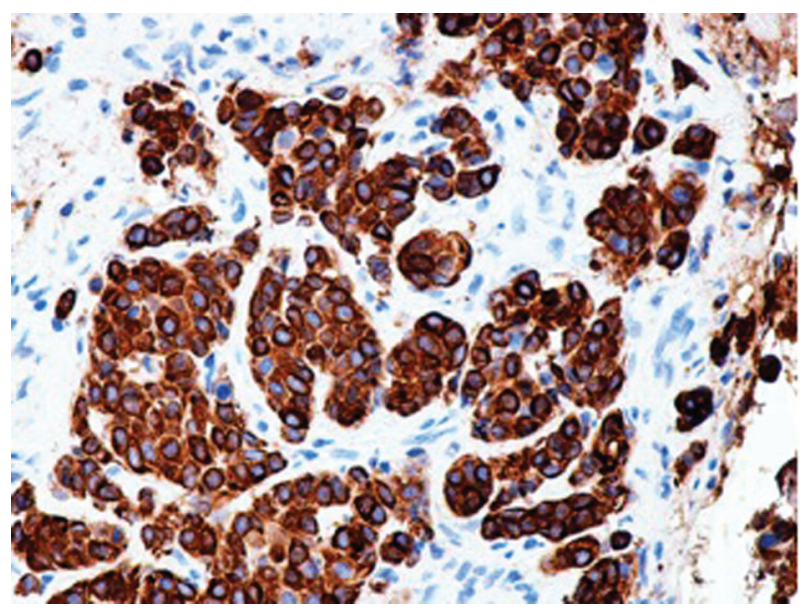

Figure 7. DAB view, hematoxylin counterstain, positive diffuse and intense reaction for CK7 in tumor cells. Anti-CK7 × 200 .

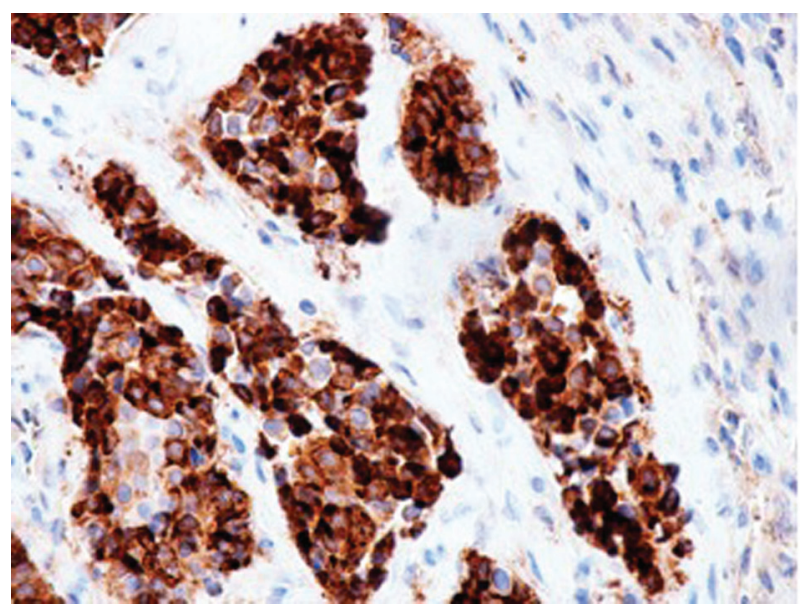

Figure 8. DAB view, hematoxylin counterstain, diffuse and intense reaction for mammaglobin. Anti-mammaglobin, $\times 200$.

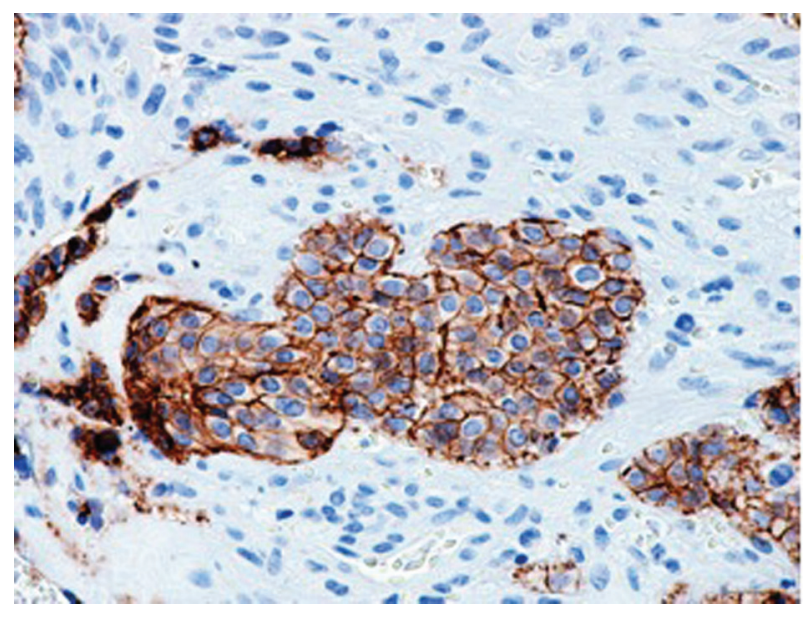

Figure 9. DAB view, hematoxylin counterstain, membrane marking for E-cadherin in tumor cells. Anti-E-cadherin, $\times 400$.

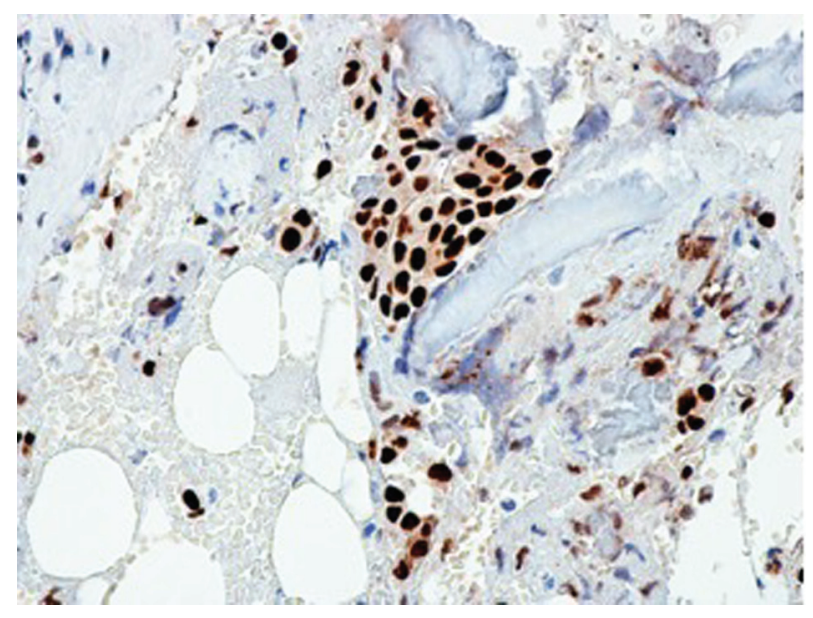

Figure 10. DAB view, hematoxylin counterstain, diffuse and intense expression of ER in tumor cell. Anti-ER, $\times 400$.

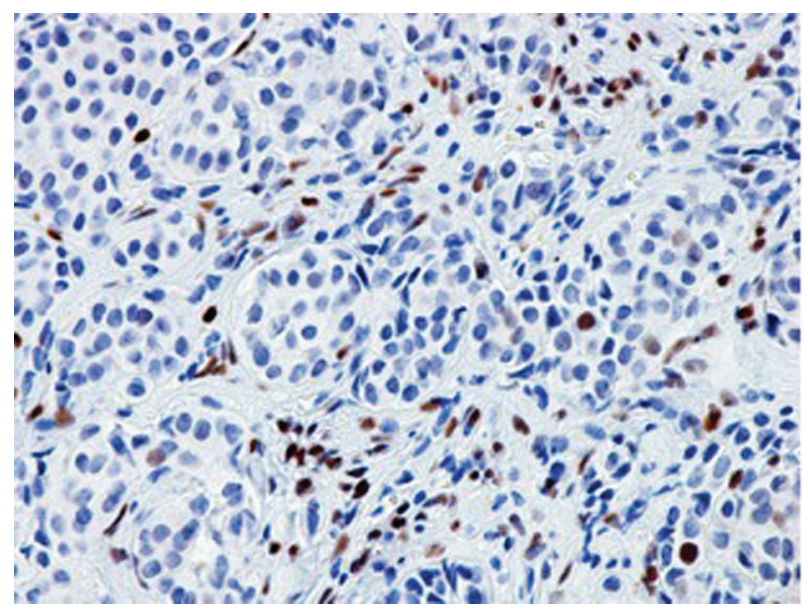

Figure 11. DAB view, hematoxylin counterstain, focal reactivity for $p 63$ in tumor cells, frequent stromal reactive cells. Anti-p63, $\times 200$. 
bined with mammography can detect the illness in the early stage when treatment is more efficient and there is a likelihood of a cure $[15,16]$.

We consider this case of a young woman with previously undetected metastatic breast cancer (although she was regularly followed by her primary care physician) and one SRE in the form of a pathological bone subtrochanteric fracture as an interesting development of the underlying pathology since she had none of the usual symptoms. As the literature shows these cases are quite rare [10-12]. There is also the aspect of surgical management, in this case we selected fixation with a reamed CMD, an implant inserted with a minimally invasive endosteal technique that allows early postoperative weight-bearing. No attempts were made to isolate or resect the metastatic tumor. While this approach may not be universally accepted, it has good literature support $[17,18]$ and some clinical advantages: it is minimally invasive, provides early and full weightbearing, has a short postoperative recovery period and allows further diagnostic tests to be performed and oncological treatment, while leaving options open for further surgical management, if necessary.

\section{Competing Interests}

The author(s) declare that they have no competing interests.

\section{Abbreviations}

3D: three-dimensional; ER: anti-estrogen receptor; CK7: anticytokeratin; CMD: cephalomedullary device; CT: computer tomography; IHC: immunohistochemistry; SRE: skeletal-related event

\section{References}

1. Rizzoli R, Akesson K, Bouxsein M, Kanis JA, Napoli N, Papapoulos S, Reginster JY, et al. Subtrochanteric fractures after long-term treatment with bisphosphonates: a European Society on Clinical and Economic Aspects of Osteoporosis and Osteoarthritis, and International Osteoporosis Foundation Working Group Report. Osteoporos Int. 2011;22(2):373-390.

2. Yoon RS, Hwang JS, Beebe KS. Long-term bisphosphonate usage and subtrochanteric insufficiency fractures: a cause for concern? J Bone Joint Surg Br. 2011;93(10):1289-1295.

3. Murphy CG, O'Flanagan S, Keogh P, Kenny P. Subtrochanteric stress fractures in patients on oral bisphosphonate therapy: an emerging problem. Acta Orthop Belg. 2011;77(5):632-637.

4. Pazianas M, Kim SM, Yuen T, Sun L, Epstein S, Zaidi M. Questioning the association between bisphosphonates and atypical femoral fractures. Ann N Y Acad Sci. 2015;1335:1-9.

5. Altaf FJ, Mokhtar GA, Emam E, Bokhary RY, Mahfouz
NB, Al Amoudi S, Al-Gaithy ZK. Metaplastic carcinoma of the breast: an immunohistochemical study. Diagn Pathol. 2014;9:139.

6. Gomes DS, Porto SS, Rocha RM, Gobbi H. Usefulness and limitations of E-cadherin and beta-catenin in the classification of breast carcinomas in situ with mixed pattern. Diagn Pathol. 2013;8:114.

7. Torre LA, Bray F, Siegel RL, Ferlay J, Lortet-Tieulent J, Jemal A. Global cancer statistics, 2012. CA Cancer J Clin. 2015;65(2):87-108.

8. Solomayer EF, Diel IJ, Meyberg GC, Gollan C, Bastert G. Metastatic breast cancer: clinical course, prognosis and therapy related to the first site of metastasis. Breast Cancer Res Treat. 2000;59(3):271-278.

9. Hussein O, Komarova SV. Breast cancer at bone metastatic sites: recent discoveries and treatment targets. J Cell Commun Signal. 2011;5(2):85-99.

10. Svendsen ML, Gammelager H, Svaerke C, Yong M, Chia VM, Christiansen CF, Fryzek JP. Hospital visits among women with skeletal-related events secondary to breast cancer and bone metastases: a nationwide population-based cohort study in Denmark. Clin Epidemiol. 2013;5:97-103.

11. Pockett RD, Castellano D, McEwan P, Oglesby A, Barber BL, Chung K. The hospital burden of disease associated with bone metastases and skeletal-related events in patients with breast cancer, lung cancer, or prostate cancer in Spain. Eur J Cancer Care (Engl). 2010;19(6):755760.

12. Felix J, Andreozzi V, Soares M, Borrego P, Gervasio H, Moreira A, Costa L, et al. Hospital resource utilization and treatment cost of skeletal-related events in patients with metastatic breast or prostate cancer: estimation for the Portuguese National Health System. Value Health. 2011;14(4):499-505.

13. Jensen AO, Jacobsen JB, Norgaard M, Yong M, Fryzek JP, Sorensen HT. Incidence of bone metastases and skeletal-related events in breast cancer patients: a population-based cohort study in Denmark. BMC Cancer. 2011;11:29.

14. Wegener B, Schlemmer M, Stemmler J, Jansson V, Durr HR, Pietschmann MF. Analysis of orthopedic surgery of bone metastases in breast cancer patients. BMC Musculoskelet Disord. 2012;13:232.

15. Anderson BO, Cazap E, El Saghir NS, Yip CH, Khaled HM, Otero IV, Adebamowo CA, et al. Optimisation of breast cancer management in low-resource and middleresource countries: executive summary of the Breast Health Global Initiative consensus, 2010. Lancet Oncol. 2011;12(4):387-398.

16. Kushi LH, Doyle C, McCullough M, Rock CL, DemarkWahnefried W, Bandera EV, Gapstur S, et al. American Cancer Society Guidelines on nutrition and physical activity for cancer prevention: reducing the risk of cancer with healthy food choices and physical activity. CA Cancer J Clin. 2012;62(1):30-67.

17. Issack PS, Barker J, Baker M, Kotwal SY, Lane JM. Surgical management of metastatic disease of the proximal part of the femur. J Bone Joint Surg Am. 2014;96(24):2091- 
2098.

18. Thambapillary S, Dimitriou R, Makridis KG, Fragkakis EM, Bobak P, Giannoudis PV. Implant longevity, compli- cations and functional outcome following proximal femoral arthroplasty for musculoskeletal tumors: a systematic review. J Arthroplasty. 2013;28(8):1381-1385. 